\title{
Quantification is Incapable of Directly Enhancing Life Quality through Healthcare*
}

\author{
Peter A. Moskovitz \\ Departments of Orthopaedic Surgery and Neurological Surgery, George Washington University, \\ Washington, DC, USA \\ Email: pmoskovitz@gmail.com
}

Received February $14^{\text {th }}$, 2013; revised March $14^{\text {th }}, 2013$; accepted March $21^{\text {st }}, 2013$

Copyright (C 2013 Peter A. Moskovitz. This is an open access article distributed under the Creative Commons Attribution License, which permits unrestricted use, distribution, and reproduction in any medium, provided the original work is properly cited.

\begin{abstract}
Quantification, the measurement and representational modeling of objects, events and relationships, cannot enhance life quality, not directly. Illustrative is Sydenham's model of disease (Sydenham, 1848-1850) and its spawn: the checklist quantification that is contained in the DSM (Diagnostic and Statistical Manual of Mental Disorders, now in its fifth edition) and ICD (International Classification of Diseases, now in its ninth edition). The use of these diagnostic catalogs is incapable of directly enhancing health, a component of life quality; because health is not the control or absence of disease, and practitioners do not think in checklists. Healthcare practitioners have adopted the methods of the airline industry in imposing checklists that are unnatural to nonlinear cognition. At first instance and finally, the subjectivity of experience and the intersubjectivity of communication create the diagnostic and therapeutic relationship that enhances health and life quality. Health is the capacity to cope and to adapt to the experience of suffering, regardless of its cause or context, and to pursue salutary experience. Society will effectively develop, implement and "reform" healthcare only when it accepts positive, noncircular definitions of health and "Quality of Life." The ethical obligation of the practitioner is to honor and trust the patient's narrative of illness, the story of suffering.
\end{abstract}

Keywords: Health; Healthcare; Quantification; Quality-of-Life; Suffering

\section{Quantification, the Measurement and Representational Modeling of Objects, Events and Relationships, Cannot Enhance Life Quality, Not Directly.}

To demonstrate how quantification enhances life quality, one assumes that quantification is, in fact, capable of enhancing life quality. Regarding the relationship of health to life quality one might begin with the assumption that health is a good and a component of life quality. Quantification enhances the delivery of healthcare, because proper diagnosis and treatment require statistical, analytical problem solving-Daniel Kahneman's “system 2" thinking (Kahneman, 2011) ${ }^{1}$. By this argument quantification enhances life quality. The conclusion is false. Assumptions grounded in the intuitively obvious are not always correct ${ }^{2}$.

I begin this essay with three assumptions that I hold as justi-

\footnotetext{
"Disclaimer: The author has no financial or competing interests to declare.

${ }^{1}$ I would be imprudent were I to omit a reference to Kahneman's important work, Thinking, fast and slow. It is exceptional in that it is both important and popular (The excellent is rarely found, more rarely valued (von Goethe, 1917)). Also, it easily comes to mind with an essay such as this. Kahneman's book is about how cognitive bias affects the apparatus of consciousness in problem solving and prediction. Deduction and objectivity, as well as induction, intuition and subjectivity are the subjects of his book. Intersubjectivity, a subject of this essay, is not among them.
}

fiable. First, “quantification” means expressing and revealing the nature of objects, events or relationships in symbolic representation, the foundation of "system 2" thinking. The symbols may be linguistic, pictorial, sculptural, structural or numerical - the media of art and science. For example, we measure the state of a person's diabetes by measuring blood sugar and A1C hemoglobin. The numbers are not the thing itself-the state of her $^{3}$ diabetes; the numbers are a symbolic representation of that disease, and an incomplete one, to be sure.

Second, life quality, or, as it is known in the literature, quality of life (QOL hereafter) is entirely subjective and incapable of precise measurement using numerical, or any other symbols. There are, of course, many instruments intended to measure QOL. They tend to be specific for the discipline for which they were developed (Costanza, 2008). That is, the social sciences, economics, medicine, psychiatry, aid organizations and development agencies use different instruments to measure QOL.

There is a general agreement that quantification of material well-being is related to QOL only in a preliminary, superficial and limited way (Kahneman \& Deaton, 2010). Quantification

\footnotetext{
${ }^{2}$ The proposition that it is both necessary and sufficient to ground one's assumptions in the intuitively obvious appears to be a staple of philosophical discourse. It qualifies as a cognitive bias (see above). I do not pretend to understand the language of philosophical discourse.

${ }^{3}$ In this essay I use the feminine pronoun to "subvert the dominant paradigm" that favors the masculine pronoun when the antecedent is not a specific person.
} 
of emotional well-being takes various forms, including, but not limited to, measurements of situational and overall "satisfaction" and states of "contentment" or "happiness". The satisfaction of healthcare consumers (patients) appears to be independent of the quality of their healthcare (Xiao \& Barber, 2008). Either there is something wrong with healthcare or there is something wrong with the quantification of "satisfaction", with the quantification of "healthcare quality" or both.

QOL, well-being, satisfaction, contentment and happiness are "irreducible concepts" (Casebeer, 2003). These expressions cannot be reduced to finite elements for measurement. Later in this essay, I introduce the expression "salutary experience", the pursuit of which is perilously close to a "pursuit of happiness". Neither can be measured with an instrument subject to external validation.

And, third, quantification as a process, an art or a method can do nothing. It is humans who might be capable of using quantification to enhance their own or another's QOL. The technology of quantification, no matter how sophisticated it is, cannot measure what is central to health, to satisfaction and to QOL, that is, the experience of it.

\section{A Negative Example of the Relationship between Quantification and Life Quality Is Sydenham's Model of Disease and Its Spawn: The Checklist Quantification Contained in the DSM (Diagnostic and Statistical Manual of Mental Disorders) and ICD (International Classification of Diseases).}

It is intuitively obvious that medical and mental disorders and diseases commonly degrade QOL. Thus, we conclude that the control and cure of disorders and diseases enhances QOL. Safe and effective treatment requires accurate diagnosis. The model of Sydenham provides for diagnostic classifications of disorders and diseases according to specific symptoms, signs, laboratory findings and imaging data (Scadding, 1988) (King, 1970) (Blashfield \& Draguns, 1996).

Stanley J. Reiser proposed that the advent of modern, scientific medicine was the moment when Laennec first used his stethoscope to listen to the sound of disease, the measurable sounds of asthmatic lungs or of a defective heart that was the cause of his patient's sickness (Reiser, 2009: pp. 6-7). The cause of illness became an object capable of measurement. The practitioner reports the measurements, shares them with others, analyzes them and studies their relationship to other objects, events and relationships. This is science, the foundation of nosology — the study of noxious events. (Sedler, 1994) (Stengel, 1959).

Practitioners measure anatomy, physiology, chemistry and function to categorize the state of a patient into specific conditions or diseases. The practitioner catalogs her perceptions of her patient's condition within the model's matrix of symptoms and signs - the objects and events of the patient's condition and the relationships among them. Nosologic catalogs, such as the DSM (American Psychiatric Association, 2005) and the ICD (Buck, (Ed.), 2013) make sense of the myriad variables that the practitioner confronts.

${ }^{4}$ Not to be confused with "irreducible complexity", which is self-contradictory. All "complexity" is reducible. (Moskovitz, 2013).

\section{The Use of Diagnostic Catalogs, such as the DSM and the ICD, Is Incapable of Directly Enhancing Health, a Component of Life Quality, because Health Is Not the Control or Absence of Disease and because Practitioners Do Not Think in Checklists.}

The World Health Organization defined "health" in 1946: "Health is a state of complete physical, mental and social wellbeing and not merely the absence of disease or infirmity" (WHO, June, 1946). The definition avoids the negative "absence of disease” by specifically excluding it. A proper definition must state what the thing is, not what it is not. But, "well-being” and "health" are synonyms. I maintain, therefore, the definition is circular. Furthermore, practitioners generally strive for the control and cure of disease, injury, and deformity; and in doing so, enhance the "well-being" of their patients. They do so, in part, because insurers who compensate healthcare practitioners do not pay for well-being. Healthcare payers compensate practitioners for either procedures (including the process of "evaluation and management") or for "health-related" outcomes, and seldom the latter. For example, the process of evaluating and treating hypertension (measuring blood pressure and prescribing medication, etc.) is easier to measure than the longitudinal outcome of lowered blood pressure over time; and, even such an "outcome measure" is an order of magnitude easier to measure than the effect of the treatment on the patient's QOL. "Health maintenance organizations” (HMO's) that appear to compensate practitioners for enhancing the well-being of their patients obtain mixed popularity (Rossiter, Langwell, Wan, \& Rivnyak, 1989), except, in my view, with healthcare economists and administrators.

Beyond the problem of how practitioners are paid, the quantification model of Sydenham fails to enhance QOL because it does not comport with how practitioners think. The encounter between patient and practitioner is not, at first, a deductive process; it is primarily inductive and immeasurable. Intuitive experience precedes deductive analysis. For the practitioner, that is as it should be. Much has been written about "how doctors think" (Groopman, 2007). I'm persuaded to think that much of it misses or obscures the point.

\section{Healthcare Practitioners Have Adopted the Methods of the Airline Industry in Imposing Checklists that are Unnatural to Nonlinear Cognition.}

Imposing linear checklists ("system 2 thinking”) upon subjectivity ("system 1") requires analytical thought. The process is necessary to prevent errors and adverse outcomes that result from the cognitive bias of induction. We measure objects, events and relationships with symbols that we manipulate with linear organization and calculation. Checklists for the diagnostic criteria that produce classifications of medical and psychiatric disorders are linear, like those that ground and flight personnel use to maintain and fly airplanes. The practitioner represents a positive finding (an object, event or relationship) on the checklist with an appropriate symbol that can be digitized. Diagnostic and therapeutic algorithms are capable of computerization and automation. Many consider this an ideal model of healthcare (Wang et al., 2003) (Agency of Health Care Policy and Research, 1996).

The practitioner decides what checklist to use, what questions to ask and how to interpret the responses to complete 
appropriate diagnostic or therapeutic algorithms ${ }^{5}$. Intuitively we understand the process as deduction, "thinking". The neurobiology of how the practitioner "thinks" about clinical problem solving may not be important to her patient. Why and when she does so, using the proper checklist, is important and the process derives from the inductive products of the narrative encounter. Deduction, and the checklists to which it applies, are properly imposed upon the practitioner-patient relationship, but that's not how the practitioner-patient encounter starts.

Practitioners don't initially think in linear algorithms capable of digitization and computational analysis by Aristotelian and Quinian logic, like the ICD and the DSM. How the practitioner thinks about her patient begins with her subjective experience, not with her linear, checklist objectivity. The perceptual, associative and motivational apparatus of the body-brain is nonlinear. Cognition, or consciousness, in or out of awareness, is nonlinear. That is, the mind is oscillatory (Crick \& Koch, 1990) (Llinas, 2001) (Singer, 2005) (Singer, 2001).

Subjective experience happens in the objective, measurable, physical body-brain by the process of reverberating, resonant, recursive oscillation of bioelectrical activity. A computer performs linear operations and calculations: electrons flow along branching pathways switched with linear controls. The physical brain, however, is capable of spontaneous and induced oscillation; and when stimuli trigger or direct oscillation among disparate circuits, networks and systems of the body-brain, consciousness happens. That is how mind happens and decisions are made. Cognitive bias occurs because the process is not under linear control.

The foregoing and what follows constitutes a theory of consciousness and, for the present case, of how practitioners think. It is, in the main, speculative, controversial, and deeply counterintuitive. The theory is not new, and my personal statement of it derives, predominantly from the work of Francis Krick and Christof Koch (Crick \& Koch, 1990), Wolf Singer (Singer, 2001), Rodolfo Llinas (Llinas, 2001) and Henry Markram (Markram, 2006). Jonah Lehrer (Lehrer, 2007), like the writers and artists about whom he wrote, along with countless others, could not accept that "mind”, "experience”, "subjectivity" and "consciousness" are synonyms, as I propose they are, and that they happen in the physical body-brain.

My interpretation of the theory proposes that the process by which we know what is "out there" and by which we know that we are certain of what we know in clinical problem-solving, or in any endeavor, is nonlinear, iterative, "dynamical" and oscillatory (Singer, 2005). The oscillatory process of mind, of consciousness is described as "emergent" (Morowitz, 2002) or "supervenient” (Chalmers, 1996). Mind “emerges” in and from the physical body-brain as oscillation. "What" oscillates enters consciousness, either in awareness or out of awareness ${ }^{6}$.

\footnotetext{
${ }^{5}$ Airlines use written checklists. Practitioners often use checklists retained in memory according to their education, training and experience. Memorized checklists, memory being nonlinear, are predictably less reliable.

${ }^{6}$ Because the non-linear, oscillatory model is not new, many neuroscientists contributed important concepts to it, e.g. Bernard Baars' "global neuronal workspace" (Baars, 1998). Still, a coherent oscillatory model has been slow to "emerge". The problem of neurophilosophy is more troublesome, in my view. An example is Paul Churchland's discussion of recursive information systems (Churchland, 2007). The medium of philosophy is language (the linear representation of thought in linguistic symbols), and, I propose, no philosophical discourse, no matter how rigorous, can equate a subjective state with its causative physical, neural event; just as philosophical discourse cannot describe what a hypercube looks like, or what are the boundaries of an imaginary number. Yet, oscillatory, iterative, chaotic systems are multidimentional and the mathematics that describes oscillatory systems invariably contains imaginary numbers (Kelso, 2006). A review of the 2500 year polemic on the nature of consciousness is not within the scope of this essay.
}

Consciousness is the subjectivity of the human condition ${ }^{7}$. What's "out there" is real, but the experience of it, like the first person experience of the redness of a red, ripe tomato (Jackson, $1982)^{8}$, or the sweetness of its taste, is unique and subjective. No one else can know what it is like for another to experience that redness. Consciousness is "the feeling of what happens", as Antonio Damasio put it (Damasio, 1994). It is the experience of "the remembered present" according to Gerald Edelman (Edelman, 1989). Consciousness is the experience of perceptual contents. Be not deceived, consciousness is not "the contents of perceptual experience" as you might read in the literature (McDowell, 1994).

Consciousness is all subjectivity without objectifiable contents. Perceptions and their bioelectrical representation have contents; our experience of them has none. John Searle understood that consciousness is "about something" but it has no objective contents. (Searle, 1983) There are no "qualia" that some philosophers think ought to be the granularity of subjective experience (Jackson, 1982). Unfortunately, Searle gave the "aboutness" of subjectivity the ungainly name of "intentionality", opposed to "extensionality". Intentionality has nothing to do with intent, and the ambiguity prompts philosophers to say "intentionality, with a capital I" to distinguish it from intentionality, meaning intended motivation in awareness 9 .

According to the present theory, consciousness is the totality of the individual's momentary experience and subjectivity. Some of it enters awareness, most of it does not, but the neurobiological process by which it happens is the same, except for the frequency of oscillation. That some perceptual contents in consciousness enter awareness while most do not is confusing, even for neurophilosophers. What enters awareness is often called "phenomenal consciousness", implying that there are other, separate realms of consciousness. Investigators call these realms of consciousness out of awareness, variously, pre-consciousness, sub-consciousness, access consciousness or subliminal consciousness. Freud initially called them collectively "the unconscious", which might be confused with Jung's characterization of experiences shared across cultures and across languages as the "collective unconscious"10.

Perceptual contents that oscillate in consciousness, whether in or out of awareness, affect behavior, affect the perception of other stimuli and how they enter consciousness and permit the storage of perceptual contents so that they can enter awareness later, when the stimulus is remote. Therefore, the theory I've presented implies that consciousness is a unitary phenomenon. Thinking and deduction that we hold so dear are no more nor less than being aware of being aware.

\footnotetext{
${ }^{7}$ This personal definition does not exclude non-human animal consciousness, which limitations in expressive capacities obscures. I infer from Markram's work (Markram, 2006) that machines can mimic consciousness but not reproduce or obtain it. The work of nanotechnology scientists, such as Alice Parker, may change that (EshaghianWilner, 2007).

${ }^{8}$ I apologized to Frank Jackson for using his coinage (Jackson, 1982) once before. I'm obliged to do so again.

${ }^{9}$ Philosophers do that sort of thing. Intentionality, being the obverse of extensionality and distinct from intent, should be spelled differently. I do not pretend to understand the language of philosophical discourse.

${ }^{10}$ The ways in which perceptual contents out of awareness affect perceptual contents in awareness is the subject of Thinking, fast and slow (Kahneman, 2011), to which I alluded earlier.
} 


\section{At First Instance and Finally, the Subjectivity of Experience and the Intersubjectivity of Communication Create the Diagnostic and Therapeutic Relationship That Enhances Health and Life Quality ${ }^{11}$.}

The reader, no doubt, asks what this speculative theory has to do with the practitioner who endeavors to understand the experience of her patient and who endeavors to enhance that patient's health, welfare and quality of life. Practitioners are interested in illness, what happens when a disease, injury or deformity evokes the experience of suffering ${ }^{12}$. Illness and suffering are states of consciousness. They evoke emotions but they are not themselves emotions, nor are they physical abnormalities of the body-brain. Suffering and illness are states of consciousness, subjective, immeasurable and incapable of being digitized, characterized or catalogued in any ICD or DSM.

Subjective experience (all experience is subjective) is immeasurable-nonlinear. And yet, consciousness feels substantive. It is "real" because it is our first person experience and ours alone. Subjectivity defines the "self", sometimes called "the autobiographical self" as if there were any other kind (Damasio, 1994). Because the "self" is subjective and existentially unique, we assiduously protect it from damage or loss. The experience of loss or threat of loss of the integrity of "self" is what we call suffering (Cassell, 2004). Scholars and scientists have tried to measure and quantify suffering and they cannot (Meyerfield, 1999) (Siu, 1993).

The practitioner serves her patient best who focuses her awareness on her patient's experience as much as, if not more than, on the objective characteristics of the patient's condition. And, the vast majority of the practitioner's experience of her patient is initially outside of her awareness. Perceptions of the patient's behavior, expressions of mood, emotion or pain, often evoke in the observer similar experience to that which evoked the patient's behavior. The process derives from the confluence of the perceptual and motor apparatus that might be called "motor mirroring", involving as it does specialized nerve cells that are called mirror neurons (Rizzolatti \& Craighero, 2005) (Oberman \& Ramachandran, 2007). The process is integral to experiences that we variously call sympathy, compassion, identification, fellow feeling and empathy (Shirtcliff, 2009). Motor mirroring begins out of awareness where, in my view, for the practitioner, it can be both a blessing and a curse ${ }^{13}$.

The practitioner first understands her patient intersubjectively ${ }^{14}$. Ultimately she does so through the practice of what has

\footnotetext{
${ }^{11}$ Cognitive bias underlies any discussion of induction and the subjectivity of how practitioners and the rest of us think. Checklists are necessary because they help to avoid error to which cognitive bias is a universal, ever-present predisposition. The problem of cognitive bias is not the subject of this essay.

${ }^{12}$ A disease, injury or deformity that does not evoke the experience of suffering may be an annoyance, but it does not rise to the level of illness or disability.

${ }^{13}$ The process of "identification" includes both perceptual and motor intersubjectivity. It is in her motivation and behavior that the practitioner must remain scrupulously separate from her patient. Empathizing with a patient is good practice, "identifying" with a patient breaches critical boundaries and is dangerous. Since empathy is subjective, one cannot teach empathy.

${ }^{14}$ Any barrier between the practitioner and the patient, such as a computer, internet consultation or the telephone, limits the capacity of both patient and practitioner to experience each other inter-subjectively. That is an important part of my "complaint”. It is only one of the many dangers of EMR-electronic medical records. These barriers are not insurmountable ones; but, unless practitioners hold them in awareness, the dangers damage both relationship and judgment.
}

come to be known as "narrative medicine" (Charon, 2006) (Kleinmann, 1988). The practitioner's understanding of her patient's experience comes from the patient's narrative of illness, the patient's story of suffering. Health, like illness, is subjective, immeasurable and narrative.

The theory presented here suggests how the practitioner focuses her awareness on experience of perceptual contents "about" her patient-multidimensional experience that is initially out of her awareness and fleeting. The difference between a linear model of consciousness and an oscillatory model of consciousness informs our understanding of the difference between the checklist mentality of evaluating and treating disease and the subjective understanding of a person's experience of that disease. I accept that there are many who hold that the former is central to good healthcare and that the latter is a luxury that modern healthcare can no longer afford-perhaps.

\section{Health Is the Capacity to Cope and to Adapt to the Experience of Suffering, Regardless of Its Cause or Context, and to Pursue Salutary Experience.}

I proposed this definition of health in collaboration with Stan Reiser. It is a positive definition, avoiding negation. Although it avoids circularity, the critical, defining expressions, i.e.: "the experience of suffering" and "salutary experience" are subjective and cannot be reduced to finite elements for measurement. It is axiomatic that subjective experience, positive or negative, is irreducible.

No objective measurement will ever demonstrate what it is like to experience illness or health. Quantification can never demonstrate what it is like to experience that redness of that red, ripe tomato, or the yellowness of a field of daffodils. Such is the nature of subjectivity. The state of a person's health is understandable only in the narrative of the experience of it.

Resilience is another component of life quality. Resilience and health, though not synonyms, inform our understanding of both. Each is a capacity. One definition of resilience ${ }^{15}$ includes the "ability [capacity] to recover readily from illness, depression, adversity, or the like; buoyancy” (Random House, 2013). “... To recover readily from... adversity..." is acceptably similar to the concept: “... to cope and adapt to the experience of suffering”. Some of the properties of resilience, such as, time and completeness of recovery, may be reduced to finite elements for measurement, as when they are used in materials science. Time and completeness of recovery from a disease or after an injury to the point of "restoring health" are not so easy to measure.

Health, like resilience, apart from being a capacity, is also a property or state. For example, one can be in a state of good health as one can have good fortune. Normative good healththat is, what constitutes the symptoms and signs of "good health" according to experts or opinion polls-might be reduced to measurable elements. The outcome of health, the experience of good health or of bad health, however, is subjective, for which quantification remains an illusion.

\section{Society Will Effectively Develop, Implement and "Reform" Healthcare Only When It Accepts Positive, Noncircular Definitions of Health and "Quality of Life".}

The Patient Protection and Affordable Care Act of 2010 (P.L.

${ }^{15}$ The first definition of resilience, "the power or ability to return to the original form, position, etc., after being bent, compressed, or stretched; elasticity”, used in materials science, is a nice metaphor for health. 
111-148) and the subsequent Healthcare and Education Reconciliation Act of 2010 (H.R. 4872) remain controversial and, in the view of many, either excessive or insufficient (DePinto, 2012). There remains a crushing irony in the debate over the legislation. The Acts are at once operational definitions of healthcare yet ones developed without a coherent and cogent definition of health. The reasons for this lapse or, at best, concession, are understandable. First, practitioners (providers) intuitively associate good health with the prevention, treatment and cure of disease, injury and deformity, as do their patients (consumers). Second, healthcare administrators and payers require documentation and accountability from providers. The documentation must be expressed in symbols that are capable of measurement, digitization and automated processing. Healthcare is dependent on quantification and a checklist mentality.

And, third, it is the natural manure of every institution that it will eventually require reform, a process that requires quantification of its ongoing state. No healthcare system will long survive if it is based only on a subjective definition of health, even if there is no scientific and ethically correct alternative. It is, therefore, inevitable that such a healthcare system will fall short of the sort of QOL enhancement that really matters, one that enhances resilience in the face of suffering and that enhances the "pursuit of salutary experience".

\section{But, Even If Health Is Erroneously Defined by Objective Process and Outcome, It Is the Ethical Obligation of Practitioners, at First Instance, to Honor and Trust Their Patients' Narratives of Illness.}

Only by hearing each patient's story of suffering in a unique personal and social context (Engel, 1997) will the practitioner understand what it is like for that patient to be ill or to be "in health" (Svenaeus, 2010). The ability to guide the diagnostic and therapeutic alliance, including the objective assessment of the patient's condition, with an intuitive, inductive and intersubjective understanding of the patient's experience separates the life-enhancing practitioner from the merely competent. Quantification cannot provide understanding of the patient's narrative. Society must choose how it will regard health and healthcare to best enhance life quality.

\section{REFERENCES}

Agency of Health Care Policy and Research (1996). Using computers to advance health care. Research in action fact sheet. AHCPR Publication No. 96-P014. Retrieved from Agency for Healthcare Research and Quality. http://archive.ahrq.gov/research/computer.htm

American Psychiatric Association (2005). Diagnostic and Statistical Manual of Mental Disorders-Fourth Edition (Text Revision). Washington, DC: American Psychiatric Association.

Baars, B. N. (1998). Neuronal mechanisms of consciousness: A relational global workspace framework. In S. K. Hameroff (ed.), Toward a Science of Consciousness II: The second Tucson discussions and debates (pp. 169-278). Cambridge, MA: MIT Press.

Blashfield, R., \& Draguns, J. (1996). Evaluative criteria for psy-chiatric classification. Journal of Abnormal Psychology, 83, 140-150.

Buck, C. J. (2013). International classification of diseases, 9th Revision, clinical modification. St. Louis: Elsevier Saunders.

Casebeer, W. (2003). Natural ethical facts: Evolution, connectionism, and moral cognition. Cambridge, MA: MIT Press.

Cassell, E. (2004). The nature of suffering and the goals of medicine.
Oxford: Oxford University Press.

http://dx.doi.org/10.1093/acprof:oso/9780195156164.001.0001

Chalmers, D. (1996). The conscious mind. Oxford: Oxford University Press.

Charon, R. (2006). Narrative medicine: Honoring the stories of illness. New York: Oxford University Press.

Churchland, P. (2007). Neurophilophy at work. Cambridge: Cambridge Univeristy Press. http://dx.doi.org/10.1017/CBO9780511498435

Costanza, R. (2008). An integrative approach to quality of life measurement, research, and policy. S.A.P.I.EN.S. 1 (1).

Crick, F., \& Koch, C. (1990). Towards a neurobiological theory of consciousness. The Neurosciences, 2, 263-275.

Damasio, A. (1994). Descartes' error: Emotion, reason and the human brain. New York: Grosset/Putnam.

Damasio, A. (1999). The feeling of what happens: Body and emotion in the making of consciousness. New York: Harcourt Brace.

DePinto, J. (2012). Public opinion of the health care law. http://www.cbsnews.com/8301-250_162-57462689/public-opinion-o f-the-health-care-law/

Edelman, G. (1989). The remembered present: A biological theory of consciousness. New York: Basis Books.

Engel, G. (1997). From biomedical to biopsychosocial: Being scientific in the human domain. Psychosomatics, 38, 521-528. http://dx.doi.org/10.1016/S0033-3182(97)71396-3

Eshaghian-Wilner, M. (2007). Emulation of Neural Networks on a Nanoscale Architecture. Journal of Physics: Conference Series, 61, 288. http://dx.doi.org/10.1088/1742-6596/61/1/058

Groopman, J. (2007). How doctors think. New York: Houghton Mifflin.

Jackson, F. (1982). Epiphenomenal aualia. The Philosophical Quarterly, 32, 127-136. http://dx.doi.org/10.2307/2960077

Kahneman, D. (2011). Thinking, fast and slow. New York: Farrar, Straus \& Giroux.

Kahneman, D., \& Deaton, A. (2010). High income improves evaluation of life but not emotional well-being. Proceedings of the National Academy of Sciences, 107, 16489-16493. http://dx.doi.org/10.1073/pnas.1011492107

Kelso, J. S. A. (2006). The complementary nature. Cambridge, MA: MIT Press.

King, L. (1970). Empiricism and rationalism in the works of Thomas Sydenham. Bulletin of the History of Medicine, 44.

Kleinmann, A. (1988). The illness narratives: Suffering, healing and the human condition. New York: Basic Books.

Lehrer, J. (2007). Proust was a neuroscientist (p. 187). New York: Haughton Mifflin.

Llinas, R. (2001). I of the Vortex. Cambridge, MA: MIT Press.

Markram, H. (2006). The blue brain project. Nature Reviews Neuroscience, 7, 153-160. http://dx.doi.org/10.1038/nrn1848

McDowell, J. (1994). The content of perceptual experience. The Philosophical Quarterly, 44, 190-205. http://dx.doi.org/10.2307/2219740

Meyerfield, J. (1999). Suffering and moral responsibility. New York: Oxford University Press.

Morowitz, H. (2002). The emergence of everything: How the world became complex. Oxford: Oxford University Press.

Moskovitz, P. (2011). Shifting realities: Myths, models \& moralityThe myth of health as the absence of disease.

http://www.nourfoundation.com/media-gallery/videos/Shifting-Reali ties-Myths-Models-and-Morality/The-Contingent-Nature-of-Reality/ Peter-A-Moskovitz-MD.html

Moskovitz, P. (2011). Understanding suffering: The phenomenology and neurobiology of the experience of illness and pain. In J. Giordano (ed.), Maldynia (pp. 33-64). Boca Raton: Taylor and Francis Group.

Moskovitz, P. (2013). Randomness does not occur in nature: Philosophical assumptions at the boundary of knowledge and certainty. Synesis.

Oberman, L., \& Ramachandran, V. (2007). The simulating social mind: The role of the mirror neuron system and simulation in the social and communicative deficits of autism spectrum disorders. Psychological Bulletin, 133, 310-327.

http://dx.doi.org/10.1037/0033-2909.133.2.310 


\section{P. A. MOSKOVITZ}

Random House (2013). Random house webster's unabridged dictionary: Indexed. New York: Random House.

Reiser, S. (2009). Technological Medicine: The changing world of doctors and patients. New York: Cambridge University Press.

Rizzolatti, G., \& Craighero, L. (2005). Mirror neuron: A neurological approach to empathy. In J. Changeux, et al. (ed.), Neurobiology of human values (pp. 107-124). Berlin: Springer-Verlag. http://dx.doi.org/10.1007/3-540-29803-7_

Rossiter, L., Langwell, K., Wan, C., \& Rivnyak, M. (1989). Patient stisfaction aong ederly erollees and disenrollees in medicare health mintenance oganizations: Results from the national medicare competition evaluation. JAMA, 262, 57-63.

http://dx.doi.org/10.1001/jama.1989.03430010069033

Scadding, J. (1988). Health and disease: What can medicine do for philosophy. Journal of Medical Ethics, 14, 118-124. http://dx.doi.org/10.1136/jme.14.3.118

Searle, J. (1983). Intentionality: An essay in the philosophy of mind. Cambridge: Cambridge University Press. http://dx.doi.org/10.1017/CBO9781139173452

Sedler, M. (1994). Foundations of the New Nosology. Journal of Medicine and Philosophy, 19, 219-238. http://dx.doi.org/10.1093/jmp/19.3.219

Shirtcliff, E. A., et al. (2009). Neurobiology of empathy and callousness: Implications for the development of antisocial behavior. Behavioral Sciences \& the Law, 27, 137-171. http://dx.doi.org/10.1002/bsl.862

Singer, W. (2001). Consciousness and the binding problem. Annals of the New York Academy of Sciences, 929, 123-146.

http://dx.doi.org/10.1111/j.1749-6632.2001.tb05712.x

Singer, W. (2005). How does the brain know when it is right? In P. Changeux, et al. (ed.), Neurobiology of human values (pp. 125-136). Berlin: Springer-Verlag. http://dx.doi.org/10.1007/3-540-29803-7_10

Siu, R. (1993). Panetics and the Dukkha: An integrated study of the infliction of suffering; The Panetics Trilogy II. Washington, DC: The International Society for Panetics.

Stengel, E. (1959). Classification of mental disorders. Bulletin of the World Health Organization, 21, 601-663.

Svenaeus, F. (2010). The Hermeneutics of medicine and the phenomenology of health: Steps towards a philosophy of medical practice. Dordrecht: KluwerAcademic Pub.

Sydenham, T. (1848-1850). Medical observations concerning the history and cure of acute disease. In R. Latham (ed.), The Works of Thomas Sydenham (p. 15). London: Sydenham Society.

von Goethe, J. (1917). Wilhelm Meister's Apprenticeship. Cambridge, MA: Harvard Classics.

Wang, S., et al. (2003). A cost-benefit analysis of electronic medical records in primary care. American Journal of Medicine, 114, 397403. http://dx.doi.org/10.1016/S0002-9343(03)00057-3

WHO (1946). Preamble to the constitution of the World Health Organization. In Official Records of the World Health Organization 2 (p. 100). New York: World Health Organization.

Xiao, H., \& Barber, J. (2008). The effect of perceived health status on patient satisfaction. Value in Health, 11, 719-725. http://dx.doi.org/10.1111/j.1524-4733.2007.00294.x 